\title{
Humanitarian Aid Workers: The Forgotten First Responders
}

\author{
Robert I.S. Macpherson, BA; ${ }^{1}$ (i) Frederick M. Burkle, Jr., MD, MPH, PhD (Hon.), DTM, FAAP, \\ FACEP $^{2}$ (1)
}

1. Unaffiliated Researcher, Charlotte, North Carolina USA

2. Professor (Ret.), Senior Fellow \& Scientist, Harvard Humanitarian Initiative, Harvard University \& T.H. Chan School of Public Health, Cambridge, Massachusetts USA

\author{
Correspondence: \\ Robert Macpherson, BA \\ 815 Mt Vernon Ave \\ Charlotte, North Carolina 28203 USA \\ E-mail: mac1kenny@gmail.com
}

Conflicts of interest: none

Keywords: humanitarian care; mental health; non-governmental agencies; posttraumatic stress disorder; war and conflict

\footnotetext{
Abbreviations:

CDC: Centers for Disease Control and Prevention

NGO: nongovernmental organization

PTSD: posttraumatic stress disorder

UN: United Nations

VA: United States Veterans Administration

Received: July 30, 2020

Accepted: August 18, 2020

doi:10.1017/S1049023X20001326

(C) The Author(s), 2020. Published by

Cambridge University Press on behalf of the

World Association for Disaster and Emergency Medicine.
}

\begin{abstract}
Humanitarian aid workers are an overlooked population within the structure of posttraumatic stress disorder (PTSD) research and assistance. This negligence is an industry-wide failure to address aid workers' psychological health issues. The suspected numbers of death by suicide, diagnosed PTSD, depression, anxiety disorders, hazardous alcohol and drug consumption, emotional exhaustion, and other stress-related problems are impossible to quantify but are considered endemic. Tools for establishing organizational frameworks for mental health and psychosocial support are readily available. However, the capacity to implement this assistance requires the creation and practice of an open and non-judgmental culture, based on the realistic acceptance that aid work has become inherently dangerous. The possibility of developing a psychological problem because of aid work has increased along with the rise in levels of disease, injury, kidnapping, and assault. As a result, expressions of traumatic stress have become the norm rather than an exception. This commentary outlines the essential steps and components necessary to meet these requirements.
\end{abstract}

Macpherson RIS, Burkle FM Jr. Humanitarian aid workers: the forgotten first responders. Prehosp Disaster Med. 2021;36(1):111-114.

\section{Introduction}

Initially, studies resulting in the clinical classification of posttraumatic stress disorder (PTSD) were associated with veterans returning from combat. A critical piece to the working diagnosis was the influence of other groups' studies, including sexual trauma survivors, holocaust survivors, and advocacy groups, such as the Lesbian, Gay, Bisexual, and Transgender (LGBT) and related at-risk communities. After the September 11, 2001 attacks on the United States and the critical engagement of first responders, subsequent analyses recognized further levels of PTSD issues that were experienced by the people who routinely deal with incidents of trauma, violence, and suffering and resulted in the population reconsidering the need to take their needs more seriously. ${ }^{1}$

However, one group of first responders remains an overlooked population within the framework of PTSD research and assistance. These women and men are humanitarian aid workers and, as a group, they experience an industry-wide failure to adequately address psychological health issues. A 2015 Guardian Global Development Professional Network (Guardian Media Group; London, United Kingdom) survey "found approximately 80\% of aid worker respondents had experienced mental health issues, with almost [one-] half declaring they were diagnosed with depression. Other studies noted $30 \%$ of aid workers returning from complex, stressful, and dangerous humanitarian environments experienced indications of PTSD." 2 The Antares Foundation (Amsterdam, The Netherlands) found this " $30 \%$ more significant than the numbers reported by the US Veterans Health Administration [VA; Department of Veterans Affairs; Washington, DC USA] of women and men who served in the Afghan War. The VA found that $11 \%$ of these veterans have a stress-related mental illness." "Additionally, the Guardian survey found many returning aid workers who only engaged in a single and short-term humanitarian event reported feeling detached, unable to fit into their former lives, and experienced changes their friends and family could not understand. ${ }^{2}$

There are approximately 450,000 professional aid workers throughout the world at any one time, many of whom move from one humanitarian emergency to another. These cycles are a form of self-medication; many aid workers find it comfortable and reassuring to be with others who share similar experiences. They have a familiar refrain, "You get addicted to this work, [because it is so] hard to settle back into normal life." 
While some humanitarian organizations recognize and address staff mental health problems, there remain significant barriers to implementing general mental health support services. Presently, assistance is mostly insufficient, stigma in organizations is still significant, and donor funding for staff wellness is inadequate, especially for national staff. ${ }^{3}$

Nearly a decade ago, the Security Management Initiative (SMI; Switzerland) produced a document entitled, "Can you get sued? Legal liability of international bumanitarian aid organizations towards their staff." It reviewed the existing laws in four European countries and America demonstrating that nongovernmental organizations (NGOs) are accountable for the same legal standards as any other group. The paper highlighted that NGOs' responsibilities for staff well-being are a legal and mandatory requirement. ${ }^{5}$ Around the same time, a series of lawsuits against aid agencies commenced on behalf of aid workers or the families of aid workers who were kidnaped, assaulted, injured, died, or suffered other violations of their person while working as humanitarians. ${ }^{6}$

In 2015, a Norwegian court found the Norwegian Refugee Council (Oslo, Norway) liable for compensation and to have acted with gross negligence in the case of Steven Dennis following his kidnapping in Kenya. Because it was the first case of its kind to reach a court ruling, it is considered a watershed moment in which organizations finally recognized they were responsible for staff well-being, including issues of psychological stress and illness suffered while providing humanitarian service. ${ }^{2}$

However, operational stresses associated with delivering humanitarian aid can be dramatic and the resulting problems involving staff mental health are seldom clear cut. Their work's physical consequences can be dangerous, but the constant ethical and moral quandaries that confront them are often cumulative. No matter how hard humanitarian workers try to handle it, constant exposure to death, starvation, and mayhem affect their belief in justice and human rights. Every personal and professional value they hold is under assault. As Romeo Dallaire writes: "[It is the] moral injuries that ravage our minds, our souls [and repeatedly] assaults ... our most sacred and fundamental values and beliefs."7

A significant problem in responding to the psychological needs of aid workers is quantifying the problem. While safety and security incidents and statistics can be measured, definitive data regarding deaths by suicide, diagnosed PTSD, depression, anxiety disorders, hazardous alcohol and drug consumption, emotional exhaustion, and other stress-related problems are difficult to gather. Aid workers still fear seeking help because of the stigma associated with mental illness or substance abuse and the potential negative impact on their career. Also, defining and implementing specific systems to support national and international staff mental health needs remain under-developed and lack attention and resources. ${ }^{8}$

\section{Creating a Framework for Mental Health and Psychosocial Support}

To establish an industry standard, the Antares Foundations and the Centers for Disease Control and Prevention (CDC; Atlanta, Georgia USA) formed a partnership with NGOs to develop a consensus approach to mitigate stress in aid workers: "The resulting Guidelines for Good Practice: Managing Stress in Humanitarian Workers was published in 2004 and revised in 2012." 3 In 2009, the United Nations (UN; New York USA) Inter-Agency Standing Committee (IASC; Geneva, Switzerland) published Guidelines on Mental Health and
Psychosocial Support in Emergency Settings. ${ }^{9}$ Additionally, in 2009, People in Aid (CHS Alliance; London, United Kingdom) "issued an extensive report on Approaches to Staff Care in International NGOs." 10

The significance of aid worker's mental health was further specified "in the European Consensus on Humanitarian Aid by the European Council [Brussels, Belgium], Parliament, and Commission's statement: Good practice in managing and supporting staff is a key management function, whereby staff safety, wellbeing, rights, dignity, and effectiveness are priorities." ${ }^{3}$ However, at the same time, the authors of the People in Aid report on Approaches to Staff Care in International NGOs emphasized, "little has been formally documented regarding the nature and extent of staff care practices across the sector." 10

Thus, whereas guidelines for psychological staff care within the humanitarian sector exist, what is missing are the practical approaches necessary to implement successful programs. The keyword is "practical." Too often, suggested frameworks seem to focus on a broad overview of implementation from a human resources director's perspective, a "top-down" approach. However, it is the aid worker who will trust their recovery and continued employment based on their trust in the organization. Consequently, the question remains: "What will instill confidence in the individual for their organization?" It must begin with a clear-cut statement defining an agency's accountability and support for staff mental health. However, that statement must be more than a memorandum distributed as a general email. Humanitarian leadership must understand how personal and daunting a mental illness is when linked to humanitarian work. The illness is devastatingly personal, and in response, the agency's leadership must convey their support in an equally personal fashion. Firstly, this requires the education of line-management on mental illnesses resulting from trauma before talking with their staff about organizational support.

Indeed, developing a culture of resilience must include protocols that incorporate the standards, policies, and guidelines to achieve the agency's goal. However, the core focus must be on an organizational commitment to its moral and ethical accountability. Two conditions must exist to ensure aid workers' acceptance of an assistance program. These are confidentiality and assurance that seeking help will not jeopardize their employment.

As such, the following components are essential to creating a trusted framework for organizational psychosocial programs from the staff members' perspective: ${ }^{11}$

- Affirm that the psychological well-being of every staff member is a primary concern;

- Include staff members from all corporate hierarchy levels in designing a support system, which builds a culture of staff well-being;

- Define clear lines of authority and procedures for providing psychological assistance;

- Determine and publish who are eligible to receive mental health support, such as permanent staff, volunteers, consultants, national staff, and part-time employees;

- Design and implement administrative procedures to safeguard confidentiality;

- Incorporate meaningful practices created to reduce and mitigate the causes of stress;

- Institute or revitalize a grievance redress mechanism;

- Identify vulnerable groups based on the complexity of their operating environment and the number of deployments; 
- Ensure the planning for psychological assistance includes the means to aid national staff;

- Provide all staff with a pre-deployment brief, including an overview of the physical and health dangers they may experience;

- Confirm ways to deliver on-site mental health assistance to staff experiencing stress in the field or appropriate and accessible telemental health services;

- Ensure aid workers receive a post-deployment brief, which includes a detailed discussion of the mental health support available and how to obtain it; and

- Ensure line managers have additional assistance, as appropriate, to manage stressful and complex situations.

These conditions should also result in promoting a culture of resilience to enhance an aid worker's ability to adjust to changing situations and help to mitigate periods of trauma and stress. This ethos goes beyond risk mitigation and traditional psychosocial support. It is a holistic approach, creating an organizational culture that values mental health by replacing negative stigma with positive reinforcement.

\section{Components of Mental Health and Psychosocial Support}

The humanitarian organization's commitment to staff psychological well-being cannot function as a separate tool, only used when a staff member suffers an emergency. Mitigating mechanisms must be planned and proactive because the demands on all humanitarian staff, both national and international, continually change and reflect the extremes of the natural environment, threat levels, and vulnerable populations' conditions. In this context, an aid agency's principal responsibility is a well-defined process for shortand long-term staff support. These protocols ensure mental health is a required inclusion in operational planning and execution.

Nevertheless, too often, even when procedures are well-defined, they are relegated to a document rather than meaningful implementation. Ultimately, they are only as good as their implementation. It is not enough to say, "we have a great set of guidelines for staff mental health;" six months later, they gather dust in a manual on someone's desk. Like any policy, but mainly an organization's commitment to mental health support, these standards must be assessed, reviewed, and updated based on feedback.

Thus, the question arises: "How do we translate those principles into a working model throughout the organization?"

It begins with an inclusive approach to building an effective system for organizational staff well-being and mental health that goes well beyond merely satisfying the mechanics of legal obligations. Aid workers must trust the process is about them, and not a means to mitigate litigation possibilities. When staff routinely confront dangerous and disquieting situations, the common element to help reduce stress is their trust and confidence in organizational leadership. Such faith begins with a demonstration that the humanitarian workforce's needs come first, and senior management is willing to share power with those who confront the complexities of aid work.

\section{Implementation Difficulties}

However, even when adequate structures are in place to assist staff, many aid workers are reluctant to seek help from their organization, often due to stereotypes and biases that result from a diagnosis of psychological illness. ${ }^{12}$ Additionally, an aid worker may face a vicious cycle. The intensity, stress, and exposure to violence may manifest as survivor's guilt or survivor's syndrome. Often, there is an assumption that those who survive the trauma and intensity of a complex humanitarian response consider themselves lucky or fortunate. However, they feel guilty for leaving their colleagues and the people they were assisting behind, analogous to how a wounded Marine feels about abandoning their unit during a conflict. The aid worker returns to a world of relative safety, with adequate food and shelter, but cannot forget the devastations of war, natural disaster, or famine.

Even within a robust organizational culture of support and resilience, aid workers may fear to acknowledge that the pressures associated with their work have caused an illness or coping difficulty. They fear the loss of their work. Beyond financial considerations, aid work tends to be a "calling." A mental illness may rob them of the opportunities that define their life and engagement in a profession they love. Thus, it is incumbent that line management is trained to recognize acute and chronic stress symptoms and have the confidence and support to intervene when necessary.

\section{The Dilemma of National Staff}

Discussions regarding the psychological issues of aid workers include references to national staff. However, they are often generalized because of cultural and legal differences, and the employment of national staff is decentralized within the structure of the host nation. This is problematic because humanitarian organizations currently employ far more national staff than expatriates, and are thus at higher risk of violence. According to The Aid Worker Security Database (Humanitarian Outcomes; London, United Kingdom):

National aid workers continue to endure most of the violence in terms of absolute numbers ... The most recent data show that while attack rates have risen for nationals and internationals, the rate increase has been steeper for national staff than for their international counterparts. Furthermore, although national and international staff now have the same overall attack rates, the fatality rates for nationals are higher than for internationals—and the gap has widened considerably in recent years. ${ }^{8}$

Although the initiatives to hire more national aid workers is commendable, it is not morally or ethically responsible for employing increasing numbers of local staff and not providing the same health and well-being benefits as enjoyed by expatriate counterparts. The Duty of Care Paper reports that approximately 50\% of international aid organizations "have systems that are not unified, not coherently implemented or not functioning properly, or have no existing unified system when it comes to national staff [mental] and physical health insurance." ${ }^{4}$

\section{Conclusions and Next Steps}

The answers to proper psychological support and care for humanitarians require solutions from staff members and the organization. At its core is the creation and practice of an open and nonjudgmental culture, based on the realistic acceptance that aid work has become inherently dangerous. The possibility of a mental health problem associated with this work is as real as the increasing possibility of disease, injury, kidnapping, and assault. As a result, forms of traumatic stress have become the norm, not an exception.

In the past several years, discussion about the future of humanitarian aid has become popular. The World Economic Forum, The Future of Humanitarian Response, World Economic Meeting 2017, and the UN The Future of Humanitarian Action describes a world where human displacement is at the highest levels in generations, and climate disasters leading to unprecedented numbers of refugees and migrants are dramatically increasing. ${ }^{13}$ Additionally, the UN has published 17 sustainable development 
goals to transform the world by $2030 .{ }^{14}$ These are noble initiatives and rightfully focus on relieving the suffering, indignity, and injustices experienced by millions of humans.

The World Economic Forum speaks to an antiquated humanitarian assistance model and the urgent need "to move the narrative from charity and aid to investing in resilience." ${ }^{33}$ The details describe necessary partnerships between governments, businesses, and humanitarian organizations; all have a common theme of moving humanitarian aid into the 21st Century. Without a doubt, these are useful and important discussions. Except one element is missing.

After years of acknowledging and describing the potential psychological effects on aid workers who carry out the assistance needed to alleviate human suffering, there remain fragmented systems based on guidelines and voluntary compliance. While professional humanitarians may have access to varying degrees of mental health support, the volunteers and consultants do their job and return to a vacuum of an organizational disinterest.

A review of existing literature shows a genuine interest and concern for aid workers' psychological health issues, but they tend to be academic and diagnostic. The Antares Foundation and CDC Guidelines for good practices to manage stress in humanitarian workers are commendable. However, like other current discussions, it does not adequately address the urgency of mental illness among aid workers. The reasons and conditions for an epidemic of aid workers' psychological trauma have been studied, analyzed, and quantified, yet humanitarians still find themselves isolated and cut off from help.

There is no fast track to creating an effective organizational mental health support service. However, it must begin by building a structure that assists staff with measures to mitigate the psychosocial risks that aid workers' experience. The first step must start with acceptance by organizational leadership that every one of their staff deployed to a crisis or spending a prolonged period in a complex or destitute situation will experience a degree of posttraumatic stress. It is not a matter of "may;" it is a reality - they will.

There must be a recognition that the organization's responsibility for staff mental health well-being is wide-ranging. Dealing with permanent national and international staff is the easy part, but what moral and legal obligation for volunteers, interns, and temporary employees?

As the aid industry attempts to define its place in the 21st Century, the first step should be for the entire community, including governmental, nongovernmental, and international organizations, to create, embrace, and guarantee a common standard, which ensures mental health needs of the women and men who confront the deprivation, disease, misery, and violence of humanitarian assistance are adequately addressed.

The existing donor requirements regarding aid workers' safety and security can provide a model to ensure organizational attention to staff psychosocial requirements. Before the US-led incursion into Iraq and Afghanistan in the early 2000s, planning for staff security was done on an organizational level. Several agencies had sound systems, but the majority paid little attention to the requirement. ${ }^{11}$

After the 2003 attack on the UN headquarters in Baghdad, and the ensuing increase in directed violence against humanitarians throughout the Middle East, governmental donors required indepth plans detailing how aid agencies would protect their staff. These procedures included the means to mitigate risks, evacuate personnel, and ensure safety, including fire plans and emergency evacuation. The plans also required an overview of crisis management procedures in the headquarters and the field. ${ }^{11}$

These plans offer an excellent means to address staff wellness. Mental health issues in a humanitarian operation are as viable and critical as the ways to mitigate threats of direct violence. Most safety and security plans contain a checklist regarding stress symptoms in a complex environment, but the attention ends within a document.

It is unfortunate, but a fact, most of the organizational attention to staff safety and security was incremental until it became a cause for litigation and a monitored and evaluated component of donor funding requirements. Currently, the systems and structures for staff mental health support exist, but as recommended guidelines through institutions such as Antares, the CDC, and the UN. However, until they are formalized and embraced throughout the humanitarian community, aid workers will continue to suffer as the "forgotten first responders."

\section{References}

1. Lewis-Schroeder NF, Kieran K, Murphy BL, Wolff JD, Robinson MA, Kaufman ML. Conceptualization, assessment, and treatment of traumatic stress in first responders: a review of critical issues. Harv Rev Psychiatry. 2018;26(4):216-227.

2. Research Suggests Mental Health Crisis among Aid Workers. The Guardian. http:// www.theguardian.com/global-development-professionals-network/2015/nov/23/ guardian-research-suggests-mental-health-crisis-among-aid-workers. Accessed June 28, 2020.

3. Managing Stress in Humanitarian Workers. Antares Foundation web site. http:// www.antaresfoundation.org/filestore/si/1164337/1/1167964/managing_stress_in_ humanitarian_aid_workers_guidelines_for_good_practice.pdf?etag $=4 \mathrm{a} 88 \mathrm{e} 3 \mathrm{afb}$ 4f73629c068ee24d9bd30d9. Accessed May 15, 2020.

4. Can you get sued? Legal liability of international humanitarian aid organizations towards their staff. Security Management Initiative (SMI), Geneva Centre for Security Policy web site. https://gisf.ngo/resource/can-you-get-sued-legal-liabilityof-international-humanitarian-aid-organisations-towards-their-staff. Accessed April $17,2020$.

5. Heintze H-J, Thielbörger P. International Humanitarian Action: NOHA Textbook. Basel, Switzerland: Springer International; 2018:8-11.

6. Yes, Aid Workers Are Getting Killed More Often. But Why? The Washington Post. https:/www.washingtonpost.com/politics/2019/12/06/yes-aid-workers-are-gettingkilled-more-often-why. Accessed May 7, 2020.

7. Dallaire RA. Waiting for First Light: My Ongoing Battle with PTSD. Toronto, Canada: Vintage Canada; 2018:175.

8. Humanitarian Outcomes. Aid Worker Security Report 2019 (updated). Humanitarian Outcomes. https://www.humanitarianoutcomes.org/sites/default/files/publications/ awsr_2019_0.pdf. Published 2019. Accessed April 3, 2020.

9. United Nations Inter-Agency Standing Committee (IASC). Guidelines for mental health and psychosocial support in emergency settings. https:/www.who.int/ mental_health/emergencies/9781424334445/en/. Published 2019. Accessed March 19, 2020.

10. People in Aid/InterHealth. Approaches to Staff Care in International NGOs. https://gisf.ngo/wp-content/uploads/2020/02/2072-InterHealth-People-in-AidApproaches-to-staff-care-in-international-ngos.pdf. Published 2009. Accessed February 17, 2020.

11. Macpherson R, Burkle FM. Neglect and failures of human security in humanitarian settings: challenges and recommendations. Prehosp Disaster Med. 2013;28(2): 174-178.

12. Surya M, JaffD, Stilwell B, Schubert J. The importance of mental well-being for health professionals during complex emergencies: it is time we take it seriously. Glob Health Sci Pract. 2017;5(2):188-196.

13. World Economic Forum. The Future of Humanitarian Response. http://www3. weforum.org/docs/WEF_The_Future_of_Humanitarian_Response.pdf. Published 2017. Accessed May 10, 2020.

14. United Nations. Sustainable Development Goals. https://www.un.org/ sustainabledevelopment/sustainable-development-goals. Published 2016. Accessed June 7, 2020. 